\title{
A influência do sedentarismo na prevalência de lombalgia
}

\author{
José Jean de Oliveira Toscano ${ }^{1}$ e Evandro Pinheiro do Egypto ${ }^{2}$
}

\section{INTRODUÇÃO}

A obtenção de equilíbrio nas estruturas que compõem a pilastra de sustentação humana (coluna vertebral), evitando quadros dolorosos a ela relacionados, não se constitui em tarefa fácil, devido principalmente às constantes mudanças de posturas realizadas diariamente pelo homem, expondo sua estrutura morfofuncional a uma série de agravos.

Um desequilíbrio mecânico das estruturas da coluna vertebral atua como fator nocivo sobre elas mesmas. Todas as estruturas que compõem a unidade anátomo-funcional do segmento lombar apresentam inervação nociceptiva, com exceção do núcleo pulposo e de algumas fibras do anel fibroso ${ }^{1}$.

As estruturas músculo-articulares são responsáveis pelo antagonismo das ações mecânicas da coluna: eixo de sustentação do corpo e, ao mesmo tempo, eixo de movimentação ${ }^{2}$. A falta ou excesso de esforço físico nessas estruturas facilmente acarretará danos à mecânica do ser humano em seus componentes osteomioarticulares.

Sucintamente, podemos definir a lombalgia como sendo um sintoma referido na altura da cintura pélvica, podendo ocasionar proporções grandiosas. O seu diagnóstico pode ser considerado simples, pois geralmente o quadro clínico da lombalgia é constituído por dor, incapacidade de se movimentar e trabalhar ${ }^{3}$.

A importância da dor lombar pode ser medida através da prevalência na população geral de adultos e em comunidades de trabalhadores, podendo manifestar-se desde a infância ${ }^{4,5}$. Evidências de problemas relacionados à coluna

1. Especialista em Ciências do Esporte; Professor do Departamento de Educação Física da Universidade Federal de Sergipe (UFS).

2. Médico Reumatologista; Professor do Departamento de Medicina Interna da Universidade Federal da Paraíba (UFPB).

Recebido em: 16/1/2001.

Aceito em: 5/6/2001.

Endereço para correspondência:

José Jean de Oliveira Toscano

Rua Otacílio de Albuquerque, 215 - Expedicionários - 58040-720 - João

Pessoa, PB

Tel.: (83) 244-9180

E-mail: jjo.toscano@bol.com.br vêm desde o período bíblico, sendo Jacó a primeira vítima referendada ${ }^{6}$.

Com o pequeno volume de dados epidemiológicos nacionais e diante da realidade nas condições de saúde relacionadas com estruturas músculo-esqueléticas, pior que a dos países do $1^{\circ}$ Mundo, alguns autores chegam a estar preocupados com indícios de uma epidemia branca, chegandose inclusive a dizer que todas as pessoas irão apresentar, pelo menos, um quadro álgico lombar em algum momento de sua vida, com a agravante de que, na maioria dos casos, a dor é de curta duração e de gravidade insuficiente para justificar uma consulta médica ${ }^{1,7,8}$.

Modernamente, a atividade física vem sendo estudada no sentido de consolidar um saber científico sobre a saúde coletiva. A vida sedentária é reconhecida, mais fortemente, como sendo importante fator contribuinte na ausência de saúde e morte precoce. A Organização Mundial da Saúde e a Federação Internacional do Esporte estimam que metade da população mundial seja inativa fisicamente ${ }^{9}$. No Brasil, cerca de $60 \%$ dos brasileiros não praticam nenhum tipo de atividade física ${ }^{10}$.

Apenas recentemente têm-se observado iniciativas quanto à aplicação de programas de exercícios físicos relacionados à promoção da saúde, sendo a grande maioria direcionada a combater agravos crônico-degenerativos de característica cardiovascular e metabólica, como as doenças do coração e obesidade; pouco esforço é despendido, ainda, em programas de atividade física relacionada à saúde, envolvendo o sistema osteomioarticular, tendo como exemplo a lombalgia.

Ao realizarmos trabalhos envolvendo a temática ${ }^{11,12}$, pudemos verificar a necessidade de discussões mais freqüentes sobre a importância do fator de risco sedentarismo na prevalência da lombalgia. O propósito deste artigo é descrever o problema da inatividade física dentro da multicausalidade de fatores de risco da lombalgia, bem como apontar a influência da aptidão física como importante fator de proteção nas síndromes lombálgicas.

\section{SEDENTARISMO E LOMBALGIA, UMA QUESTÃO DE SAÚDE PÚBLICA}

Apesar de numerosas causas e fatores de risco que estão relacionados com a lombalgia, vários pesquisadores a ca- 
racterizam como uma doença de pessoas com vida sedentária; a inatividade física estaria relacionada direta ou indiretamente com dores na coluna; a maior parte da atenção dirige-se a considerá-la um subproduto da combinação da aptidão músculo-esquelética deficiente e uma ocupação que force essa região ${ }^{13,14}$.

Num estudo longitudinal publicado recentemente ${ }^{15}$, foram estudados fatores ocupacionais relacionados com a lombalgia. Os resultados apontaram que, em ambos os sexos, tanto o sedentarismo como o trabalho com grandes cargas representam indicadores de risco para a lombalgia.

Para caracterizar determinado agravo à saúde como sendo ou não problema de saúde pública, é comum analisá-lo segundo critérios básicos: magnitude, transcendência e vulnerabilidade ${ }^{16}$.

Magnitude refere-se à abrangência, ao alcance que a doença atinge na população, o contingente de pessoas acometidas por sua ocorrência. Estudos epidemiológicos envolvendo países como os EUA, Reino Unido, Escandinávia, Canadá e Brasil atestam que dores nas costas, como condição geral, episodicamente afetam cerca de $75 \%$ da população na maioria das nações industrializadas ${ }^{14}$. Pudemos observar que $72 \%$ da população investigada em clínicas particulares de reumatologia, com diagnóstico confirmado de lombalgia, tinham característica sedentária ${ }^{12}$.

Entende-se como transcendência o custo social dos agravos à população, ou seja, tudo o que passa a interferir diretamente nas relações sociais, econômicas, profissionais e culturais; dores nas costas representam um grande fator no absenteísmo laborativo; outro fator preocupante são os dados relativos ao processo de invalidez permanente ou temporária. Quando são reunidos todos os custos relacionados à lombalgia, as despesas para os negócios, indústria e governo são estimadas em até US\$ 50 bilhões anuais ${ }^{13}$. Os prováveis benefícios do exercício com menos despesas em tratamentos médicos e dias parados no serviço, menos lesões e recuperação mais rápida em caso de acidentes, além do bem-estar do indivíduo, justificam a adesão de empresas a programas de atividades físicas para seus funcioná$\operatorname{rios}^{17-19}$.

A vulnerabilidade corresponde a quanto a doença pode ser controlada com a adoção de medidas apropriadas; a lombalgia pode resultar de uma só causa, ou de várias, e podem existir significativas correlações entre elas. A maioria das lombalgias é freqüentemente atribuída a fatores mecânicos, ou sejam, relacionados com posições inadequadas, repetitivas, assumidas no dia-a-dia, associados às deficiências musculares ${ }^{20,21}$. Músculos fracos associados ao uso inadequado no dia-a-dia expõem as estruturas da coluna a agravos; o fortalecimento da musculatura do tronco pode proporcionar maior proteção nas atividades diárias ${ }^{22}$.
O programa de exercício físico pode contribuir para amenizar a carga de trabalho, tolerar melhor o estresse postural e proteger de alguns perigos no trabalho manual ${ }^{23}$.

Em recente estudo ${ }^{24}$, os autores propõem um modelo de prognóstico da lombalgia não apenas baseado em fatores clínicos associados com a dor, mas também através de variáveis do estado pré-mórbido, sendo a persistência dos sintomas associada a baixos níveis de atividade física.

No dizer de Dever ${ }^{25}$, o modelo epidemiológico tradicional, originário de um discurso centralizado, envolvendo agente, hospedeiro e ambiente, começou a ser substituído por um novo modelo epidemiológico, denominado conceito de campo de saúde, abrangendo: ambiente, estilo de vida, biologia humana e sistema de organização de cuidados. Baseando-se nesse conceito e cientes de que saúde não é um processo estático, o grande desafio atual seria organizar as tarefas do cotidiano, tendo como preocupação o alinhamento postural nas diversas atividades e o desenvolvimento dos componentes da aptidão física, com fins a ter uma condição biológica adequada de proteção contra os agravos crônico-degenerativos que o estilo de vida moderno nos proporciona, podendo aproveitar melhor o ambiente que nos cerca.

No âmbito da saúde coletiva, o compartilhamento de enfoques e perspectivas entre as várias áreas do conhecimento, principalmente nos profissionais da saúde, deve ser uma constante. De acordo com Carneiro ${ }^{26}$, se a melhoria de vida da população está cimentada em uma concepção cooperada de conhecimentos empíricos e teóricos, parece evidente que não há como excluir o processo de interdisciplinaridade entre esses diferentes tipos e níveis de conhecimento; e acrescenta que um saber com fundamentação unidimensional é uma forma de ignorância instituída.

A prática de atividades físicas na prevenção e reabilitação de lombalgias estabelece uma relação positiva já defendida por Hipócrates, quando o mesmo reconhecia o valor dos exercícios físicos para o fortalecimento dos músculos débeis ${ }^{27}$. Vislumbra-se o exercício como potencial ferramenta no que diz respeito às questões de saúde pública, notadamente pela prevenção de agravos e pela redução do custo de tratamentos. A educação física pode contribuir de forma importante na prevenção de síndromes dolorosas na coluna por proporcionar, através de programas de força e flexibilidade, maior conscientização da postura ${ }^{28}$.

Em atitude oposta ao sedentarismo, a atividade física esportiva, com fins competitivos, oferece riscos de dores e doenças nas costas associadas com esforços musculares acentuados e repetitivos ${ }^{5,29,30}$. Por outro lado, a atividade praticada sem orientação adequada pode representar problemas. Pudemos observar em nosso estudo ${ }^{12}$, com a amostra constituída de não-atletas, de ambos os sexos, que, nos 
indivíduos praticantes de atividades físicas com diagnóstico de lombalgia, a freqüência era de $40 \%$ para a caminhada, sendo seguida do futebol, $26,7 \%$, e do voleibol, $13,4 \%$.

Em particular, enfatiza-se a relação da lombalgia com a fraqueza dos músculos paravertebrais e, sobretudo, abdominais, e nos baixos níveis de flexibilidade na região lombar e dos grupos musculares da parte posterior da coxa, bem como em atividades físicas de contato e impacto ${ }^{5,30}$.

\section{A INFLUÊNCIA DA APTIDÃO FÍSICA NA SAÚDE LOMBAR}

Não é difícil observar que níveis irrisórios de aptidão física são comuns no estilo de vida sedentário e em algumas atividades profissionais, nas quais raramente há necessidade de esforços físicos. Níveis adequados de aptidão física podem contribuir na postura corporal durante as funções diárias com economia de energia sem exceder o limite tolerável músculo-articular ${ }^{17}$.

Apesar de haver evidências de que grupos ativos têm menor probabilidade de sofrer lesão e dor na região lombar, ou seja, menos episódios que seus congêneres sedentários $^{32}$, apenas o fato de realizar uma atividade física no cotidiano não implica fator de proteção para a coluna ${ }^{33}$, sendo os cuidados com o tipo de exercício, nível de atividade, carga de trabalho, postura corporal na infância/adolescência merecedores de criteriosos cuidados ${ }^{34}$.

Os componentes da aptidão física que podem ser apontados como "indicadores de saúde" são aqueles que oferecem alguma proteção contra o aparecimento de distúrbios orgânicos provocados pelo estilo de vida sedentário, portanto, extremamente sensível ao nível de prática de atividade física: resistência cardiorrespiratória, composição corporal, força, resistência muscular e flexibilidade ${ }^{35}$.

Apesar de cientes da associação positiva do peso corporal (gordura) e dor na coluna lombar e da recomendação em preveni-la através dos componentes resistência cardiorrespiratória e composição corporal ${ }^{36,37}$, iremos direcionar nossa discussão em relação às valências força e flexibilidade, por acreditarmos que baixos níveis desses componentes representam expressiva incidência de distúrbios músculo-esqueléticos na coluna lombar ${ }^{38}$.

Sendo os movimentos da coluna resultantes de uma série de pequenos deslocamentos de ossos e tecidos moles, atuando sob a ação de músculos, sem esses, ela seria totalmente instável, pois sua função é manter a postura. Teoricamente, músculos fracos atingem a condição isquêmica e de fadiga mais facilmente que músculos fortes, aumentando as probabilidades de lesões e dificultando manter a coluna em seu alinhamento adequado. Um estudo japonês demonstrou que indivíduos com uma história prévia de lom- balgia apresentam pouca força muscular no tronco e fraqueza muscular generalizada, se comparados com aqueles que não apresentam essa patologia ${ }^{39}$.

Déficits de força muscular associada a lombalgias crônicas ocorrem em função de que a atrofia muscular resultante leva a sobrecarga de outras estruturas lombares, bem como a diminuição da coordenação do correto movimento a ser realizado pelas estruturas osteomioarticulares, nos esforços de levantamento de peso nas atividades diárias?. Estudo com escolares identificou menor força dos músculos da coluna e do abdome nos indivíduos que sentiam dor na lombar comparados com os controles ${ }^{40}$.

Quanto à flexibilidade, indivíduos inativos apresentam amplitude articular reduzida em várias tarefas diárias; o resultado é, normalmente, rigidez articular e limitação grave do movimento, impossibilitando-o de ser realizado sem dor ${ }^{17}$.

A melhora da amplitude do movimento tem sido associada com alívio dos sintomas nas lombalgias agudas e crônicas, podendo ser observada em muitos programas de tratamento e reabilitação; o papel de níveis elevados de flexibilidade associados a menos riscos de futuras queixas lombares não apresenta ainda sólida base científica ${ }^{41}$.

Portanto, pessoas mais fracas necessitam de mais esforços para realizar determinadas tarefas, ficando mais expostas a lesões, e pessoas pouco flexíveis, em geral, têm dificuldade de manter as várias posturas, estressando os discos vertebrais.

$\mathrm{O}$ equilíbrio muscular da força e da flexibilidade dos músculos agonistas e antagonistas não implica indicar que os dois segmentos devem ter semelhantes índices de força e flexibilidade. Não podemos esquecer da variabilidade individual, em que a avaliação dos testes motores e necessidade funcional de desempenho no cotidiano é que irá apontar a correta prescrição desses componentes. Em geral, as pessoas apresentam a região da musculatura do abdome mais fraca e da coluna menos flexível ${ }^{32}$.

Nossa vivência em academias de ginástica faz-nos apontar, como importante fator de proteção à coluna, exercícios resistidos associados a alongamentos específicos. As queixas de indivíduos sedentários com dores ocasionais nessa região, quando reavaliados num período médio de 90 dias, dificilmente demonstravam as inquietações anteriores relativas à coluna. Temos, como exemplo importante, um estudo de caso $^{11}$, no qual um indivíduo do sexo feminino, após dois anos de cirurgia de hérnia de disco e devida reabilitação médico-fisioterápica, realizou um trabalho de condicionamento físico conosco e obteve expressiva melhora nos componentes força, resistência muscular, flexibilidade e composição corporal, além da mudança em seu comportamento, aumentando em muito sua auto-estima e segurança. 
A aplicação de exercícios resistidos para o desenvolvimento das valências físicas força e resistência muscular localizada tem na sala de musculação seu principal meio de desenvolvimento. À medida que o músculo é progressivamente sobrecarregado, através de exercícios com pesos devidamente orientados e controlados, a força, resistência à fadiga, ou ambas, irão aumentar, sendo o risco de lesões diminuído. Estudo comparando levantadores de peso e trabalhadores industriais demonstraram que aqueles, avaliados por radiografia, tiveram menor incidência de degeneração nos discos vertebrais ${ }^{42}$.

É possível que o próprio trabalho com pesos torne a pessoa mais forte, mas as probabilidades de lesões na prática da musculação existem, principalmente no período de adaptação e na utilização de peso livre ${ }^{43,44}$, ou seja, o não controle do volume e intensidade dos exercícios pode ser mais um agente contribuindo para desordem na coluna.

$\mathrm{Na}$ elaboração de um programa de exercícios com fins de proteção à coluna, seria importante fortalecer e alongar os membros superiores e inferiores, dada a sua colaboração para levantar, conduzir pesos, diminuindo a utilização da força na coluna. Contudo, de nada adianta exercitar-se quando a postura não é observada nas atividades durante todo o dia, inclusive no dormir ${ }^{45}$.

Com os números de prevalência de lombalgia sempre expressivos a favor de diversos segmentos da população, talvez mais importante que prevenir o aparecimento da dor lombar é evitar que ela volte; se não forem tomadas providências quanto à mudança de hábitos, os riscos de recidiva aumentam $60 \%$ no mesmo ano ou no seguinte, sendo a falta de exercícios um dos fatores que causam a cronicidade das dores na coluna ${ }^{3}$.

Algumas indicações são de que baixos níveis de aptidão músculo-esquelética são fatores premonitórios da lombalgia recorrente, com o fortalecimento dos grupos musculares que protegem a coluna; apesar de não se poder prevenir futuras lombalgias, seguramente o número de episódios será menor $^{28}$. Em nosso estudo ${ }^{12}$, dos indivíduos com lombalgia recorrente, que visitaram o médico pelo menos uma vez, 93,7\% eram caracterizados como sedentários.

Por ser a lombalgia uma síndrome de característica multicausal, seu tratamento ideal demonstra ser complexo, apresentando grande variedade de opiniões entre os médi$\cos ^{46}$. Quando a etiologia é associada a fatores mecânicos, pudemos constatar certa unanimidade entre os especialistas na eficácia da prática de exercícios de fortalecimento e alongamento do aparelho locomotor ${ }^{12}$. $\mathrm{O}$ grande problema do médico em indicar seu paciente para um programa de exercícios numa academia de ginástica, por exemplo, está associado ao pouco relacionamento deste com profissionais de educação física, ocasionando insegurança em re- comendar tal procedimento. Por outro lado, é uma realidade o limitado interesse e conhecimento dos profissionais que avaliam e prescrevem atividades motoras objetivando o sentido amplo da prevenção (primário, secundário, terciário) em academias.

Apesar dos elementos favoráveis à aptidão músculo-articular na saúde lombar apresentados até o momento, o mais prudente seria afirmar que as evidências ainda são limitadas e que as intervenções dos exercícios podem representar, ainda que discretamente, diminuição da incidência e duração dos episódios de lombalgia ${ }^{47,48}$.

\section{CONCLUSÃO}

Grande parte dos profissionais de saúde, contrariando a literatura recente sobre prescrição de exercícios relacionados à saúde ${ }^{13,49}$, tem como parâmetro exclusivo de trabalho e avaliação dos níveis de saúde, incluindo a lombar, a capacidade aeróbia ${ }^{50-52}$. De acordo com Gaya e Andrade ${ }^{53}$, os músculos e articulações são elementos tão relevantes como as fontes energéticas que os mobilizam.

A elaboração de programas educativos de prevenção e recorrência da lombalgia através da atividade física deve ser direcionada no sentido de desencadear conhecimentos, atitudes e comportamentos compatíveis com uma dinâmica social fisicamente ativa e desenvolvida no quotidiano de vida, ao longo da existência das pessoas, independente da sua área de atuação. E, principalmente, em reduzir a cultura do medo instalada na maioria das pessoas em praticar atividades físicas ${ }^{54}$, em especial exercícios com peso.

Embora, correntemente, a relação aptidão física/lombalgia seja direcionada na óptica dos respectivos benefícios, as investigações ainda são insuficientes e deficitárias, dificilmente encontrando fundamentações convincentes.

De modo geral, a relação custo-benefício do exercício físico na promoção da saúde justifica o abandono imediato do sedentarismo, sendo perfeitamente promissora a implementação de programas de exercícios nos mais diversos segmentos da população. As evidências são favoráveis aos indivíduos com melhores níveis de aptidão física, no sentido de manterem menor dispêndio de esforço físico em determinada carga de trabalho.

\section{REFERÊNCIAS}

1. Cecin HA, Molinar MHC, Lopes MAB, Morickochi M, Freire M, Bichuetti JAN. Dor lombar e trabalho: um estudo sobre a prevalência de lombalgia e lombociatalgia em diferentes grupos ocupacionais. Rev Bras Reumatol 1991;31:50-6.

2. Knoplich J. Enfermidades da coluna vertebral. $2^{\underline{a}}$ ed. São Paulo: Panamed, 1986.

3. Bernard C. Lombalgia e lombociatalgias em medicina ocupacional. Revista Brasileira de Medicina 1993;50:3-9. 
4. Mendes R. O impacto dos efeitos da ocupação sobre a saúde dos trabalhadores: I. Morbidade. Rev Saúde Pública 1988;22:311-26.

5. Troussier B. Back pain in school children, a study among 1178 pupils. Scand J Rehabil 1994;26:143-56.

6. Mercúrio R, Chagas JCM, Oliveira CEAS, et al. Lombalgia. Jornal Brasileiro de Medicina 1993;64:71-8.

7. Cheren AJ. A coluna vertebral dos trabalhadores: alterações da coluna relacionadas com o trabalho. Arquivos Catarinenses de Medicina 1992; 21:139-48.

8. Wells N. Back pain. Office of Health Economics 1985;78:3-36.

9. WHO/FIMS. Exercise for health. Bull World Health Organ1995;73:1356.

10. Folha de S. Paulo. Datafolha: $60 \%$ dos brasileiros estão parados. Especial: Mexa-se. São Paulo: p. 12, 27/11/1997.

11. Toscano JJO. Os exercícios de musculação na reabilitação pós-cirúrgica de hérnia de disco. Sprint Magazine 1996;85:44-8.

12. Toscano JJO, Egypto EP. Frequiência da prática de exercícios físicos em indivíduos com diagnóstico de lombalgia em clínicas de reumatologia. Anais do XXI Simpósio Internacional de Ciências do Esporte, São Paulo, 8 a 11 de outubro, p. 130, 1998.

13. Nieman DC. Exercício e saúde. São Paulo: Manole, 1999.

14. Santos AC. O exercício físico e o controle da dor na coluna vertebral. Rio de Janeiro: Medsi, 1996.

15. Thorbjornsson CB, Alfredsson L, Fredriksson K, Michelsen H, Punnet L, Vingard E, et al. Physical and psychosocial factors related to low back pain during a 24 -year period. A nested case-control analysis. Spine 2000;25:369-74.

16. Gonçalves A, Araújo Jr B, Matiello Jr E, Ghirotto FMS, Fatarelli IF, Ayres SG. Lesões desportivas: O quê? Com quê? Por quê? In: Gonçalves A (org.). Saúde coletiva e urgência em educação física. Campinas, SP: Papirus, 1997.

17. Achour Jr A. Estilo de vida e desordem na coluna lombar: uma resposta dos componentes da aptidão física relacionada à saúde. Revista Brasileira de Atividade Física e Saúde 1995;1:36-56.

18. Cady LD, Bischoff DP, O'Connell ER, Thomas PC, Allan JH. Strength and fitness and subsequent back injuries in firefighters. Journal of Occupational Medicine 1979;21:269-72.

19. Shepard RJ. Current perspectives of the economics of fitness and sport with particular reference to worksite programmes. Sports Med 1989; 7:286-309.

20. Antônio SF, Szajubok JCM, Chahada WH. Lombalgias e lombociatalgias: como diagnosticar e tratar. Revista Brasileira de Medicina 1995;52. Especial:85-102.

21. Bankoff ADP, Moraes AC, Galdi EHG, Pellegrinotti IL, Moreira ZW. Alterações morfológicas do sistema locomotor decorrentes de hábitos posturais associados ao sedentarismo. Anais do XIX Simpósio Internacional de Ciências do Esporte. São Paulo, outubro, p. 96, 1994.

22. Santarém JM. Musculação: princípios atualizados. São Paulo: Fitness Brasil, 1995.

23. Troup JDG. Biomechanics of the vertebral column. Physiotherapy 1979; 65:238-44.

24. Thomas E, Silman AJ, Croft PR, Papageorgion AC, Jayson MI, MacFarlane GJ. Predicting who develops chronic low back pain in primary care: a prospective study. BMJ 1999;318:1662-7.

25. Dever GEA. A epidemiologia na administração dos serviços de saúde. São Paulo: Pioneira, 1988.

26. Carneiro MA. Interdisciplinaridade na pós-graduação. In: Anais do Simpósio "Interdisciplinaridade em questão". Universidade Estadual da Paraíba, Campina Grande, PB, 1998;14-20.
27. Roig JLG, Baglanz IC, Sanchez HM, et al. Los ejercícios de Williams en el tratamiento del dolor lumbosacro. Revista Cubana de Ortopedia y Traumatologia 1990;4:46-53.

28. Kisner C, Colby LA. Exercícios terapêuticos: fundamentos e técnicas. $2^{\underline{a}}$ ed. São Paulo: Manole, 1992.

29. Ferguson RJ, McMaster JH, Stanistski CL. Low back pain in college football linemen. J Sport Med 1974;2:63-9.

30. Sward L, Hellstrom M, Jacobsson BO, Peterson L. Back pain and radiologic changes in the thoracolumbar spine of athletes. Spine 1990;15: 124-9.

31. Pollock LM, Wilmoore JH. Exercícios na saúde e na doença. $2^{\underline{a}}$ ed., Rio de Janeiro: Medsi, 1993.

32. Flax HJ. Exercises for low back pain syndromes. Bol Asoc Med P R 1986;78:539-40.

33. Ueno S, Hisanaga N, Jonas H, Shibata E, Kamijina M. Association between musculoskeletal pain in Japanese construction workers and job, age, alcohol consumption, and smoking. Ind Health 1999;37:449-56.

34. Harreby M, Nygaard B, Jessen T, Larsen E, Storr A, Fisker L. Risk factors for low back pain in a cohort of 1389 Danish school children: an epidemiologic study. Eur Spine J 1999;8:444-50.

35. Bohme MTS. Aptidão física, aspectos teóricos. Revista Paulista de Educação Física 1993;7:52-65.

36. Deyo RA, Bass JE. Life-style and low back pain: the influence of smoking and obesity. Spine 1989;14:501-6.

37. Mellin G. Correlation of spinal mobility with degree of chronic low back pain after correction for age and anthropometric factors. Spine 1988;13: 173-8.

38. Saudek CE, Palmer KA. Back pain revisited. J Orthop Sports Phys Ther 1987;8:566-566.

39. Lee J-H, Ooi Y, Nakamura K. Measurement of muscle strength of the trunk and the lower extremities in subjects with history of low back pain. Spine 1995;20:1994-6.

40. Salminen JJ. Spinal mobility and trunk muscle strength in 15 year-old school children with and without low back pain. Spine 1992;17:405-11.

41. Bigos $S$. The role of spinal flexibility in back pain complaints with industry, a prospective study. Spine 1990;15:768-73.

42. Locke JC. Stretching away from back pain injury. Occup Health Saf 1983;52:8-13.

43. Mundt DJ, Kelsey JL, Golden AL, Panjabi MN, Pastides H, Berg AT, et al. An epidemiologic study of sports and weight lifting as possible risk factors for herniated lumbar and cervical discs. Am J Sports Med 1993; 21:854-60.

44. Oliva OJ, Bankoff ADP, Zamai CA. Possíveis lesões musculares e/ou articulares causadas por sobrecarga na prática da musculação. Revista Brasileira de Atividade Física \& Saúde 1998;3:15-23.

45. Caillet R. Lombalgias: síndromes dolorosos. $3^{3}$ ed. São Paulo: Manole, 1988.

46. Cherkin DC, Deyo RA, Wheeler K, Ciol MA. Physician views about treating low back pain: the results of a national survey. Spine 1995;20: 1-10.

47. Lahad A, Malter AD, Berg AO, Deyo RA. The effectiveness of four interventions for the prevention of low back pain. JAMA1994;272:128691.

48. Croft PR, Papageorgiou AC, Thomas E, MacFarlane GJ, Silman AJ. Short-term physical risk factors for new episodes of low back pain. Prospective evidence from the South Manchester Back Pain study. Spine 1999;24:1556-61.

49. Pate RR, Pratt M, Blair SN, Haskel WL, Macera CA, Bouchard C, et al. Physical activity and public health: a recommendation from the Centers

Rev Bras Med Esporte - Vol. 7, № 4 - J ul/ Ago, 2001 
for Disease Control and Prevention and the American College of Sports Medicine. JAMA 1995;273:402-7.

50. Wittink H, Hoskins T, Wagner A, Sukiennik A, Rogers W. Deconditioning in patients with chronic low back pain: fact or fiction? Spine 2000; 2221-8.

51. Sjolie AN. Access to pedestrian roads, daily activities, and physical performance of adolescents. Spine 2000;25:1965-72.
52. Feverstein M, Berkowitz SM, Huang GD. Predictors of occupational low back disability: implications for secondary prevention. J Occup Environ Med 1999;41:1024-31.

53. Gaya A, Andrade B. Os aspectos metodológicos para o desenvolvimento das capacidades condicionais em sedentários e coronariopatas. Espaço 1993;1:67-76.

54. Keen S, Dowell AC, Hurst K, Tovey P, Williams R. Individuals with low back pain: how do they view physical activity? Fam Pract 1999; 16:39-45. 